\title{
Serological diagnosis of echinococcosis: the diagnostic potential of native antigens
}

\author{
Schweiger, A ; Grimm, F ; Tanner, I ; Müllhaupt, B ; Bertogg, K ; Müller, N ; Deplazes, P
}

\begin{abstract}
PURPOSE: Human alveolar (AE) and cystic echinococcosis (CE) caused by the metacestode stages of Echinococcus multilocularis and E. granulosus, respectively, lack pathognomonic clinical signs. Diagnosis therefore relies on the results of imaging and serological studies. The primary goal of this study was to evaluate the efficacy of several easy-to-produce crude or partially purified E. granulosus and E. multilocularis metacestode-derived antigens as tools for the serological diagnosis and differential diagnosis of patients suspicious for AE or CE. METHODS: The sera of 51 treatment-naïve AE and 32 CE patients, 98 Swiss blood donors and 38 patients who were initially suspicious for echinococcosis but suffering from various other liver diseases (e.g., liver neoplasia, etc.) were analysed. RESULTS: According to the results of enzyme-linked immunosorbent assays (ELISA), metacestode-derived antigens of E. granulosus had sensitivities varying from 81 to $97 \%$ and $>99.9 \%$ for the diagnosis of CE and AE, respectively. Antigens derived from E. multilocularis metacestodes had sensitivities ranging from 84 to $91 \%$ and $>99.9 \%$ for the diagnosis of $\mathrm{CE}$ and AE, respectively. Specificities ranged from 92 to $>99.9 \%$. Post-test probabilities for the differential diagnosis of AE from liver neoplasias, CE from cystic liver lesions, and screening for AE in Switzerland were around 95, 86 and 2.2\%, respectively. Cross-reactions with antibodies in sera of patients with other parasitic affections (fasciolosis, schistosomosis, amebosis, cysticercosis, and filarioses) did occur at variable frequencies, but could be eliminated through the use of confirmatory testing. CONCLUSIONS: Different metacestode-derived antigens of E. granulosus and E. multilocularis are valuable, widely accessible, and cost-efficient tools for the serological diagnosis of echinococcosis. However, confirmatory testing is necessary, due to the lack of species specificity and the occurrence of cross-reactions to other helminthic diseases.
\end{abstract}

DOI: https://doi.org/10.1007/s15010-011-0205-6

Posted at the Zurich Open Repository and Archive, University of Zurich ZORA URL: https://doi.org/10.5167/uzh-59877

Journal Article

Originally published at:

Schweiger, A; Grimm, F; Tanner, I; Müllhaupt, B; Bertogg, K; Müller, N; Deplazes, P (2012). Serological diagnosis of echinococcosis: the diagnostic potential of native antigens. Infection, 40(2):139-152.

DOI: https://doi.org/10.1007/s15010-011-0205-6 


\title{
Serological diagnosis of echinococcosis: the diagnostic potential of native antigens
}

\author{
A. Schweiger $\cdot$ F. Grimm $\cdot$ I. Tanner $\cdot$ \\ B. Müllhaupt $\cdot$ K. Bertogg $\cdot$ N. Müller $\cdot$ \\ P. Deplazes
}

Received: 20 April 2011/Accepted: 27 September 2011/Published online: 11 November 2011

(C) Springer-Verlag 2011

\begin{abstract}
Purpose Human alveolar (AE) and cystic echinococcosis (CE) caused by the metacestode stages of Echinococcus multilocularis and E. granulosus, respectively, lack pathognomonic clinical signs. Diagnosis therefore relies on the results of imaging and serological studies. The primary goal of this study was to evaluate the efficacy of several easy-to-produce crude or partially purified E. granulosus and E. multilocularis metacestode-derived antigens as tools for the serological diagnosis and differential diagnosis of patients suspicious for $\mathrm{AE}$ or $\mathrm{CE}$.

Methods The sera of 51 treatment-naïve AE and $32 \mathrm{CE}$ patients, 98 Swiss blood donors and 38 patients who were initially suspicious for echinococcosis but suffering from various other liver diseases (e.g., liver neoplasia, etc.) were analysed.

Results According to the results of enzyme-linked immunosorbent assays (ELISA), metacestode-derived antigens of E. granulosus had sensitivities varying from 81 to $97 \%$ and $>99.9 \%$ for the diagnosis of $\mathrm{CE}$ and $\mathrm{AE}$, respectively. Antigens derived from E. multilocularis metacestodes had sensitivities ranging from 84 to $91 \%$ and $>99.9 \%$ for the diagnosis of $\mathrm{CE}$ and $\mathrm{AE}$, respectively. Specificities ranged from 92 to $>99.9 \%$. Post-test
\end{abstract}

\footnotetext{
A. Schweiger · F. Grimm (ه) - I. Tanner · P. Deplazes Institute of Parasitology, University of Zurich, Winterthurerstrasse 266a, 8057 Zurich, Switzerland e-mail: felix.grimm@uzh.ch

B. Müllhaupt $\cdot$ K. Bertogg

Division of Gastroenterology and Hepatology,

University Hospital of Zurich, Zurich, Switzerland

N. Müller

Institute of Parasitology, University of Bern, Bern, Switzerland
}

probabilities for the differential diagnosis of AE from liver neoplasias, CE from cystic liver lesions, and screening for $\mathrm{AE}$ in Switzerland were around 95, 86 and 2.2\%, respectively. Cross-reactions with antibodies in sera of patients with other parasitic affections (fasciolosis, schistosomosis, amebosis, cysticercosis, and filarioses) did occur at variable frequencies, but could be eliminated through the use of confirmatory testing.

Conclusions Different metacestode-derived antigens of E. granulosus and E. multilocularis are valuable, widely accessible, and cost-efficient tools for the serological diagnosis of echinococcosis. However, confirmatory testing is necessary, due to the lack of species specificity and the occurrence of cross-reactions to other helminthic diseases.

Keywords Echinococcosis - Diagnosis - Serology · Metacestode

\section{Introduction}

Human alveolar (AE) and cystic echinococcosis (CE) are caused by the metacestode (larval) stages of the fox tapeworm Echinococcus multilocularis and the dog tapeworm Echinococcus granulosus, respectively. Humans are infected by orally ingesting viable parasite eggs. Endemic areas for $\mathrm{AE}$ are restricted to the temperate and arctic regions of the northern hemisphere, and the disease is widespread in parts of Central Europe, Asia, and North America [1]. The liver is the most common site of $\mathrm{AE}$ lesions, and the parasite behaves in a way similar to a malignant tumour, showing both local invasive and metastatic growth, although the growth rate is much slower than in neoplasia. The treatment of AE is based on surgery in 
combination with anthelminthic treatment for at least 2 years or, in inoperable cases, on lifelong chemotherapy [2]. Untreated, mortality is high with a 10- and 15-year survival rate of 29 and $0 \%$, respectively [3]. However, with state-of-the-art treatment, prognosis is favourable, and the life expectancy of patients is almost normal [4]. In contrast, CE occurs almost worldwide, except for some regions of Central Europe and North America. In addition to the liver, the lungs and bones are also frequently involved in the disease. Unlike in AE, the parasite shows no invasive behaviour but grows as a clearly demarcated space-occupying lesion. However, spreading may occur after the spontaneous, traumatic or iatrogenic rupture of cysts (e.g. intraperitoneal). Growth of the cysts mainly leads to compression of the organs affected and may interfere with organ function leading to unspecific symptoms. Also, CE does not inevitably lead to death if left untreated [5]. Treatment is based on surgery or other invasive methods in combination with anthelminthic treatment that is aimed at the total eradication of viable parasite material [2].

Several recent reports suggest that incidences and prevalences of $\mathrm{AE}$ and $\mathrm{CE}$ are increasing in various parts of the world. For example, in Switzerland, the incidence of AE increased more than twofold from 2001-2005 compared to previous years (1993-2000: mean incidence 0.10 cases/100,000 inhabitants per year; 2001-2005: 0.26 cases/ 100,000 inhabitants per year [6]). In Lithuania, there were 10-16 reported AE cases/year in 2002-2006 compared with 0-4 cases/year in 1997-2001 [7]. Several reports have also highlighted increasing numbers of cases in Asia. In Kyrgyzstan, the incidence of CE has increased from 5.4 in 1991 to 18 in 2000 [8]. Several regions endemic for CE as well as AE have been recognised in north-western, central and north-eastern China, and AE has also spread to the Japanese island of Hokkaido in the last decades [9, 10].

As $\mathrm{AE}$ and $\mathrm{CE}$ have no pathognomonic features, their diagnosis relies heavily on the results of imaging studies and serological methods [2]. Current guidelines for the diagnosis of $\mathrm{AE}$ and $\mathrm{CE}$ are primarily based on clinical and epidemiological findings compatible with AE/CE. In addition, definitions of cases are based on the results of species-specific serological tests and imaging studies, with positive results of either one enabling a possible diagnosis of $\mathrm{AE} / \mathrm{CE}$, and positive results for both allowing a probable diagnosis of AE/CE. The gold standard for confirmation of cases is histopathological evaluation and/or the detection of parasite-specific DNA or parasite material (e.g. protoscoleces in $\mathrm{CE}$ ) in bioptic samples [2].

The most widely used antigens for the serological diagnosis of $\mathrm{AE}$ and $\mathrm{CE}$ are crude or partially purified native antigens isolated from metacestode stages of both species. A number of recombinant antigens have also been developed, but their use in standard diagnostic laboratories is limited [11]. Immunofluorescence antibody tests (IFAT), based on protoscoleces of E. granulosus, have been in use since 1967 [12] and can have diagnostic sensitivities of $>95 \%$ in hepatic CE but suffer from relatively poor specificities [2].

The protoscolex-derived antigens of E. multilocularis as well as E. granulosus, which are used in enzymelinked immunosorbent assays (ELISA) or immunoblots, have also been studied. Auer et al. [13] described the value of antigens derived from supernatants of $E$. multilocularis protoscoleces maintained in vitro. In immunoblot assays, these antigens showed no cross-reactivity to sera of patients suffering from cysticercosis, schistosomosis or fasciolosis and were even able to differentiate $\mathrm{AE}$ from $\mathrm{CE}$ in all 40 cases studied [13]. In two other studies, affinity-purified E. multilocularis metacestode antigens Em2 or Em2(G11) [14, 15] showed substantially improved species specificities of $>95 \%$ and allowed the serological identification of $\mathrm{AE}$ in a considerable number of cases.

Common crude antigens derived from E. multilocularis metacestode material, including protoscoleces, contain various amounts of Em2, a very immunogenic compound of metacestode tissue (mainly from the laminated layer of cyst walls) $[15,16]$. To assess the diagnostic performance of novel, more standardised antigens, removal of Em2 and cyst wall material is mandatory.

As native antigen, cyst fluid contains numerous lipoand glycoproteins, salts, carbohydrates and lipids, as well as some components derived from the host, such as albumin and immunoglobulins; consequently, the composition of these antigens is subject to variability depending on the stage of the cyst as well as the corresponding host [17-19]. Thus, differences in diagnostic performance in terms of sensitivity, specificity and crossreactivity have to be expected. Nevertheless, a comparative analysis by Lorenzo [20] and colleagues of the diagnostic potential of $\mathrm{EgHF}$ from six different batches applied on the same sera displayed differences in the intensity of the reaction, but there was no impact on the diagnostic parameters.

In contrast, the composition of purified protoscoleces is likely to be less dependent on the stage of cysts and contains fewer host-derived-proteins, theoretically, therefore yielding a higher reproducibility of results as a step towards further standardisation of the serological diagnosis of echinococcosis.

The aim of the this study was to evaluate the diagnostic value of different crude and partially purified native E. multilocularis- and E. granulosus-derived antigens, which can be prepared without sophisticated technical equipment and to assess their diagnostic potential in different defined clinical settings. 


\section{Materials and methods}

Sera

Fifty-one sera of treatment-naïve AE patients (sample taken prior to treatment initiation) from the University Hospital of Zurich (USZ) Echinococcosis Cohort as well as 32 sera from CE patients (29 treatment-naïve, three relapses) were used. Diagnosis was achieved in all patients according to the World Health Organisation (WHO) criteria [2]. Sera of clinically and/or parasitologically diagnosed patients suffering from amoebic liver abscess (19 patients), cysticercosis (19), fasciolosis (16), filarioses (8) and schistosomosis (18) were used for the evaluation of cross-reactions. Cut-off values were determined using sera of 98 Swiss blood donors. Thirty-eight sera of patients with other pathologies affecting the liver but an initial clinical suspicion for echinococcosis from the USZ Department of Gastroenterology (16 liver cysts, 2 hepatitis cases, 13 neoplasias, 1 sarcoidosis, 1 peliosis hepatis, 1 sclerosing cholangitis, 1 liver cirrhosis and 3 unknown causes despite extensive clinical evaluation) were used in the evaluation of specificities and likelihood ratios.

\section{Patient characteristics}

\section{AE patients}

Of the 51 treatment-naïve $\mathrm{AE}$ patients, 29 were female and 22 were male; the average age was $54 \pm 16$ years. Most patients were of Swiss nationality (48/51). Three patients had immigrated into Switzerland more than 24 years previously. The liver was involved in all patients, and seven patients had involvement of other organs [lung (3), diaphragm (2), spleen (1), adrenal gland (1), kidney (1), intraperitoneal (1)].

\section{CE patients}

Of the $32 \mathrm{CE}$ patients, ten were female and 22 were male; the average age was $35 \pm 15$ years. Most patients originated from the Balkans [Macedonia (6 patients), Kosovo (2), Albania (1), Montenegro (1)]. It was not possible to determine the exact allocation of three other patients originally from countries of the former Yugoslavia. Eight patients were from Turkey, two each were from Portugal and Italy, and one each was from Iraq and Uruguay. Only five patients had lived in Switzerland all of their life, and two of these had near relatives in Kosovo. Cysts were localised in the liver (20 patients), liver and lung (2), liver and peritoneal cavity (2), liver and bone (1), lung (1), skeleton (3) and abdomen (3).
Liver cysts were classified according to the WHO classification system [21] based on ultrasound (US) findings as follows: CE1 (4 patients), CE2 (4), CE3 (5), CE5 (3) and extrahepatic (7); the results of imaging studies were not available for nine patients.

Parasite isolates

Echinococcus multilocularis metacestode tissue (isolate IPZ CH Hd2, derived from a Swiss dog) was maintained in gerbils (Meriones unguiculatus) by repetitive intraperitoneal transplantation, as described elsewhere [22]. For preparation of E. multilocularis vesicular fluid, metacestode tissue was cultivated in vitro, resulting in the formation of metacestode vesicles, as described by Müller and colleagues [23]. Fertile E. granulosus cysts were collected from sheep in Kyrgyzstan. An overview of the parasite isolates used and important steps in the production of the different antigens is given in Table 1.

\section{Preparation of cyst fluid antigens (EmVF, EgHF)}

Echinococcus multilocularis metacestode vesicles were harvested from in vitro cultures, washed three times in phosphate buffered saline (PBS) and sliced. Vesicle fluid in PBS was used as the antigen (EmVF). For preparation of E. granulosus hydatid fluid (EgHF) antigens, fluid from fertile cysts was aspirated. Both the aspirate and harvested vesicle fluid were centrifuged at $14,000 \mathrm{~g}$ for $20 \mathrm{~min}$ at $4{ }^{\circ} \mathrm{C}$ and then dialysed against PBS for $48 \mathrm{~h}$.

\section{Preparation of cyst/Em2-depleted E. multilocularis} protoscolex antigen $(\mathrm{EmP})$

Echinococcus multilocularis metacestode material was mechanically reduced to small pieces and repetively washed in PBS. After passage through a $150-\mu \mathrm{m}$ sieve as a first purification step, protoscoleces were retained in an $80-\mu \mathrm{m}$ sieve [29], washed twice in PBS and further purified from small cysts and debris of the laminated layer containing the Em2-antigen using magnetic beads (Dynabeads; Rat anti-Mouse IgG1; Invitrogen Dynal AS, Oslo, Norway) loaded with monoclonal antibodies specific for Em2 (mAb Em2G11) [15]. In brief, $25 \mu \mathrm{l}$ of Dynabeads were washed twice in PBS, following which $7.5 \mu \mathrm{l}$ of mAb Em2G11 was added and the Dynabeads/ $\mathrm{mAb}$ Em2G11 incubated at room temperature for $30 \mathrm{~min}$. This was followed by the addition of $1 \mathrm{ml}$ of a protoscolex solution and further incubation on ice for $30 \mathrm{~min}$. After separation in a magnetic field for $2 \mathrm{~min}$, cystdepleted protoscoleces were harvested from the supernatant. The EmP antigen was prepared as described for 
Table 1 Overview of primary materials and antigen preparation processes of crude or partially purified native antigens

\begin{tabular}{|c|c|c|c|}
\hline Antigen & Primary material & Species and morphological correlate & Preparation \\
\hline $\mathrm{EgHF}$ & Hydatid cyst, sheep & Echinococcus granulosus cyst fluid & Centrifugation, dialysis \\
\hline $\mathrm{EgP}$ & Hydatid cyst, sheep & E. granulosus whole protoscolex & $\begin{array}{l}\text { Freezing at }-198^{\circ} \mathrm{C} \text {, thawing, ultrasonication, } \\
\text { centrifugation, dialysis }\end{array}$ \\
\hline EgPI & Hydatid cyst, sheep & E. granulosus protoscolex integument & $\begin{array}{l}\text { Freezing at }-20^{\circ} \mathrm{C} \text {, thawing, sedimentation } \\
\text { centrifugation, dialysis }\end{array}$ \\
\hline EmVF & $\begin{array}{l}\text { Echinococcus multilocularis } \\
\text { metacestode cultivated in vitro }\end{array}$ & E. multilocularis vesicular fluid & Cutting, centrifugation, dialysis \\
\hline EmP & $\begin{array}{l}\text { E. multilocularis metacestode } \\
\text { grown in gerbils }\end{array}$ & $\begin{array}{l}\text { E. multilocularis whole protoscolex; } \\
\text { Em2/cyst wall-depleted }\end{array}$ & $\begin{array}{l}\text { Harvesting of protoscoleces by sieving; cyst- } \\
\text { depletion by } \mathrm{mAb} \text { Em } 2 \mathrm{G} 11 \\
\text { Freezing at }-198^{\circ} \mathrm{C} \text {, thawing, ultrasonication, } \\
\text { centrifugation, dialysis }\end{array}$ \\
\hline EmPI & $\begin{array}{l}\text { E. multilocularis metacestode } \\
\text { grown in gerbils }\end{array}$ & $\begin{array}{l}\text { E. multilocularis protoscolex } \\
\text { integument; Em2/cyst wall-depleted }\end{array}$ & $\begin{array}{l}\text { Harvesting of protoscoleces by sieving; Em2- } \\
\text { depletion by } \mathrm{mAb} \text { Em } 2 \mathrm{G} 11 \\
\text { Freezing at }-20^{\circ} \mathrm{C} \text {, thawing, centrifugation, dialysis }\end{array}$ \\
\hline $\mathrm{EmC}$ & $\begin{array}{l}\text { E. multilocularis metacestode } \\
\text { grown in gerbils }\end{array}$ & E. multilocularis whole metacestode & $\begin{array}{l}\text { Freezing at }-198^{\circ} \mathrm{C} \text {, thawing, homogenisation, } \\
\text { ultrasonication, centrifugation, dialysis }\end{array}$ \\
\hline
\end{tabular}

E. granulosus protoscoleces. Cyst-depleted antigen at a high concentration of $0.01 \mathrm{mg} / \mathrm{ml}$ gave negative results in a mAb Em2G11-based sandwich ELISA [15].

Preparation of E. granulosus protoscolex antigens $(\mathrm{EgP})$

Protoscoleces were harvested from cysts, sieved and washed as described above, frozen/thawed three times in liquid nitrogen and a water bath $\left(37^{\circ} \mathrm{C}\right)$. After ultrasonication and centrifugation $\left(14,000 \mathrm{~g}\right.$ for $20 \mathrm{~min}$ at $\left.+4^{\circ} \mathrm{C}\right)$, the supernatant containing soluble proteins was dialysed against PBS and used as antigen.

\section{Preparation of integument antigens (EmPI, EgPI)}

Protoscoleces of E. granulosus and E. multilocularis (cyst/ Em2-depleted, as described above) were frozen at $-20^{\circ} \mathrm{C}$ and thawed. After sedimentation for $5 \mathrm{~min}$, the supernatant was centrifuged at $14,000 \mathrm{~g}$ for $3 \mathrm{~min}$ at $4^{\circ} \mathrm{C}$. Soluble integument constituents in the supernatant were dialysed against PBS for $48 \mathrm{~h}$ at $4^{\circ} \mathrm{C}$ (EgPI, EmPI).

Preparation of E. multilocularis metacestode antigens (EmC)

Metacestode material was collected from infected gerbils. For preparation of crude antigen fractions the material was homogenised in PBS with a disruptor prior to freezing/ thawing at $-198^{\circ} \mathrm{C}$, ultrasonication $(40 \% / 30 \mathrm{~W} / 3 \times 20 \mathrm{~s}$. $)$, centrifugation $\left(14,000 \mathrm{~g}\right.$ for $30 \mathrm{~min}$ at $\left.4^{\circ} \mathrm{C}\right)$ and dialysis against PBS.
Defined antigens

Antigen B (AgB) was prepared as described elsewhere [24, 25], the Em2(G11) antigen was prepared as described by Deplazes et al. [15], and the recombinant Em18 antigen was provided by the Institute of Parasitology in Bern, Switzerland [26, 27].

Enzyme-linked immunosorbent assay

All ELISA were performed in 96-well microtiter plates (Nunc; Maxisorp, Roskilde, Denmark). Protein concentrations were measured by the Bradford method (Bio-Rad Protein Assay; Bio-Rad, Hercules CA). Optimal antigen concentrations are known based on the results of previous titration experiments (data not shown). Plates were coated with the respective antigen in $0.1 \mathrm{M}$ carbonate/bicarbonate buffer ( $\mathrm{pH}$ 9.6) containing $0.02 \% \mathrm{NaN}_{3}$ and left standing overnight at $4{ }^{\circ} \mathrm{C}$. The plates were then washed four times with physiological $\mathrm{NaCl} / 0.3 \%$ Tween- 20 (NaCl-T) and saturated for $30 \mathrm{~min}$ at $37^{\circ} \mathrm{C}$ with $\mathrm{PBS}(\mathrm{pH}$ 7.2) containing $0.02 \%(\mathrm{w} / \mathrm{v}) \mathrm{NaN}_{3}, 0.05 \%(\mathrm{w} / \mathrm{v})$ bovine haemoglobin and $0.3 \%$ (v/v) Tween-20 (PBS-T). A 100- $\mu$ l aliquot of sera diluted 1:200 in PBS-T was added per well and incubated for $1 \mathrm{~h}$ at $37^{\circ} \mathrm{C}$. The plates were then washed four times with $\mathrm{NaCl}-\mathrm{T}$, and $100 \mu$ lof anti-human-immunoglobulin G (IgG; polyclonal rabbit anti-human-IgG, specific for the $\mathrm{C}_{\mathrm{H}} 2$ domain; DAKO, Glostrup, Denmark) conjugated to alkaline phosphatase (Roche Applied Science, Rotkreuz, Switzerland) at a dilution of 1:500 in PBS-T was added. After incubation for $1 \mathrm{~h}$ at $37^{\circ} \mathrm{C}$, the plates were washed five times $(\mathrm{NaCl}-\mathrm{T})$, and $100 \mu \mathrm{l}$ per well of a $1 \mathrm{mg} / \mathrm{ml}$ solution of 
p-nitrophenyl phosphate disodium salt hexahydrate (cat. no. 71768; Sigma, St. Louis, MO) in $0.05 \mathrm{M}$ carbonate/bicarbonate buffer ( $\mathrm{pH} 9.8$ ) containing $1 \mathrm{mM} \mathrm{MgCl}_{2}$ was added. Absorbance values were read at $405 \mathrm{~nm}\left(\mathrm{OD}_{405}\right)$ with a reference wavelength of $630 \mathrm{~nm}$. Positive standard sera of human patients with parasitologically proven $\mathrm{AE}$ or $\mathrm{CE}$ and negative standard sera of blood donors were included in all test runs. For the determination of cut-off-values, receiveroperator-curve (ROC) analyses were performed [28]. For all antigens, except the species-specific antigens Em2G11 and Em18, Swiss blood donors were defined as negative sera, whereas sera of $\mathrm{AE}$ and $\mathrm{CE}$ patients were defined as positive sera. For the Em2G11 and Em18 antigens, Swiss blood donors and $\mathrm{CE}$ patients were taken as negative sera and $\mathrm{AE}$ patients as positive sera. Cut-off values were set at the $\mathrm{OD}_{405}$ corresponding to the maximum Youden Index (defined as: sensitivity + specificity -1 ) [29].

Immunoelectrotransfer blot

Confirmatory tests for $\mathrm{CE}$ by $\mathrm{AgB}$ enzyme-linked immunoelectrotransfer blot (AgB-EITB) were performed as described elsewhere [24].

\section{Evaluation of test performances}

Sensitivities for all antigens were evaluated in terms of their efficacy in the serological diagnosis of $\mathrm{AE}$ or CE. Specificities were evaluated for Swiss blood donors and patients with non-parasitic liver lesions or parasitic infections.

Likelihood ratios and post-test probabilities (reviewed in [30]) were calculated for different clinical and epidemiological settings:

1. Differential diagnosis of $\mathrm{AE}$ from liver neoplasias (Swiss patients). The prevalence of $\mathrm{AE}$ among all patients presenting with imaging studies compatible with liver neoplasia or $\mathrm{AE}$ was assumed to be $1.5 \%$ : AE incidence in Switzerland (0.26, [6]) divided by the incidence of the most common primary liver cancer cases (cholangiocarcinoma and hepatocellular carcinoma; incidence 7.7; Source: National Statistical Office of Switzerland; available online: http://www. bfs.admin.ch) and liver metastases of colon-carcinoma (incidence of metastasis of 10 [31]).

2. Differential diagnosis of CE from liver cysts (Swiss patients). CE in Swiss patients presenting with cystic liver lesions in imaging studies was estimated at $1 \%$ on the basis of figures available for the University Hospital Zürich [abdominal US, chest computed tomography (CT), chest X-rays: 200,000 per year, CE cases: 2-5 per year; KB, personal communication].
3. The annual incidences (2001-2005) of probable or confirmed AE cases in Switzerland were 0.26 [6]. As it is known from animal experiments that serological test scores are positive as early as $4-8$ weeks post-infection [32] and due to the long asymptomatic period of 5-15 years in AE [2,3], the given incidence would not reflect the prevalence of cases that would become clinically relevant within the following 15 years but which already score positive in the serological screening. Thus, the annual incidences were multiplied by 15 as a rough estimate of the actual prevalence of asymptomatic individuals who are already infected, adding up to a prevalence of $0.042 \%$ in all of Switzerland.

As no further clinical data were known and no studies on the sensitivity/specificity of differentiating $\mathrm{AE}$ or $\mathrm{CE}$ from their most common differential diagnoses based on imaging study results were available, the prevalences specified above were used as pre-test probabilities for further calculations.

Statistical analyses

Statistical analysis and ROC were performed in SPSS ver. 17.0 (SPSS, Chicago, IL).

Binomial confidence intervals for sensitivity, specificity and predictive values were calculated according to the Clopper and Pearson method. The McNemar test for associated binominal values was used to calculate significance within patient groups (e.g. comparison of sensitivity for $\mathrm{AE}$ in separate antigens).

\section{Results}

Diagnostic performance: sensitivities of single tests

The sensitivities of the tests [with 95\% confidence intervals (CIs)] for the serological diagnosis of either $\mathrm{AE}$ or $\mathrm{CE}$ were calculated for all antigens and are summarised in Table 2.

All crude or partially purified antigens had very high sensitivities in testing for $\mathrm{AE}(\geq 99.9 \%$, 95\% CI 94.3-100\%) regardless of the parasite species they had been prepared from. The results were not significantly different from those of the most widely used standard EgHF-ELISA. AgB, Em2(G11) and the Em18 antigen detected $\geq 99.9 \quad(95 \%$ CI $94.3-100), \quad 88.2 \quad$ (95\% CI $76.1-95.6$ ) and $92.2 \%$ (95\% CI $81.1-97.8 \%$ ) of the cases, respectively (differences not significant).

Sensitivities of the crude or partially purified antigens for $\mathrm{CE}$ ranged from 81.3 (95\% CI 63.6-92.8, EmVF) to 


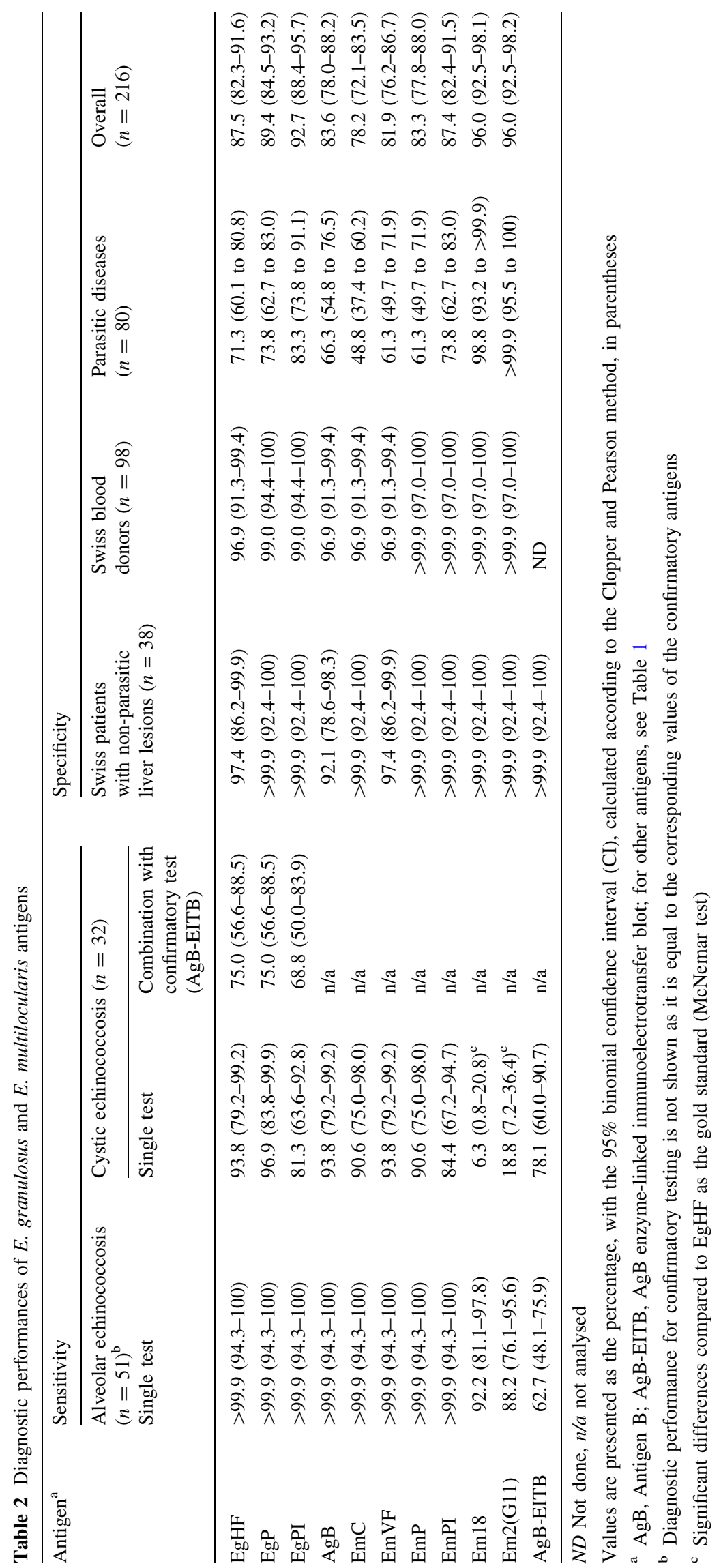


96.9\% (95\% CI 83.8-99.9\%; EgP). AgB used in ELISA had a sensitivity of $93.8 \%$ (95\% CI 79.2-99.2\%). Again, the differences were statistically not significant, when compared to the those of the EgHF-ELISA. AgB-EITB had a sensitivity of 62.7 (95\% CI 48.1-75.9) and 78.1\% (95\% CI 60.0-90.7\%) for the diagnosis of $\mathrm{AE}$ and $\mathrm{CE}$, respectively.

Of the seven CE patients without hepatic involvement, only one patient with an isolated lesion in the omentum would have been missed (EgPI-ELISA). False negative reactions in cases with liver involvement occurred in two inactive or transitional stage cysts and one active stage cyst with the use of EgPI (CE3: 1/5; CE5: 1/3; CE3: 1/5; CE1: 1/4) and four inactive or transitional stage cysts with the use of AgB-EITB (CE3: 3/5; CE5: 1/3). However, no significant difference was found. In two patients scoring false negative when $\mathrm{EgHF}, \mathrm{EgP}, \mathrm{EgPI}$ and AgB-ELISA, respectively, were used, the cyst stage could not be evaluated.

Diagnostic performance: species-specific diagnosis and confirmatory testing

With the exception of the purified Em2(G11) and recombinant Em18 antigen, all antigens tested reacted with serum antibodies from most AE and CE patients and therefore did not allow for a species-specific serological diagnosis. In the ELISA based on the most species-specific antigens [Em18 and Em2(G11)], positive reactions with antibodies in the serum samples from CE patients in the range of $6.3 \%$ $(0.8-20.8 \%)$ and $18.8 \%(7.2-36.4 \%)$, respectively, were recorded. The combined sensitivity for AE of both tests (positive was defined as a patient scoring positive in either test) was 98.0 (range 89.6-100.0) with cross-reactions to $\mathrm{CE}$ in the range of $18.8 \%(7.2-36.4 \%)$. As all crude or partially purified antigens reached sensitivities of $>99.9 \%$ in our evaluation, the sensitivities and values for the crossreacting $\mathrm{CE}$ sera of the Em2(G11)- and Em18-based tests also represent the diagnostic performance of combined testing for AE with the use of one of these two antigens in a confirmatory test. The diagnosis of $\mathrm{CE}$ with confirmation by AgB-EITB reached sensitivities ranging from 68.8 (95\% CI 50.0-83.9; EgPI) to 75\% (95\% CI 56.6-88.5\%; several antigens) (see Table 2). With respect to the localisation and cyst stages, AgB-EITB failed to recognise one patient with a bone lesion, three patients suffering from CE3 and one patient with type CE5 cysts.

Diagnostic performance: specificity and cross-reactions

Specificities were calculated for 38 patients with nonparasitic liver lesions from Switzerland, 80 patients with other parasitic infections and 98 Swiss blood donors (Table 2). Specificities estimated for patients with nonparasitic liver lesions were excellent, ranging from 92.1 (95\% CI 78.6-98.3; AgB-ELISA) to $>99.9 \%$ (95\% CI 92.4-100\%). Differences, when compared to the results of the standard EgHF-ELISA, were not significant. Specificities for the AgB-EITB, the Em2G11- and Em18ELISA were $>99.9 \%(92.4-100 \%)$ for patients with non-parasitic liver lesions.

Cross-reactions with antibodies in sera of 80 patients suffering from different other parasitic infections were found at varying extents in all antigens and are summarised in Table 3. Among the crude or partially purified antigens, the EgPI antigen proved to be most specific (13 false positive reactions). The most common cross-reactions were recorded with antibodies present in the sera of cysticercosis and fasciolosis patients. However, tests based on the species-specific antigens Em2(G11) and Em18 gave no false positive reactions for these two patient groups. Hardly any cross-reactions with antibodies in the sera of patients with amoebic liver abscesses were recorded. Only one crossreaction with amoebic liver abscess was recorded for the recombinant Em18 antigen, and the Em2G11 and AgBEITB antigens exhibited no cross-reactions at all.

Table 3 Cross-reactions to antibodies in sera of patients suffering from selected parasitic infections

\begin{tabular}{|c|c|c|c|c|c|c|c|c|c|c|c|}
\hline \multirow[t]{2}{*}{ Parasitic disease } & \multicolumn{10}{|c|}{ Number of false positive reactions } & \multirow[t]{2}{*}{ AgB-EITB } \\
\hline & $\mathrm{EgHF}$ & $\mathrm{EgP}$ & EgPI & $\mathrm{AgB}$ & EmC & EmP & EmPI & EmVF & $\operatorname{Em} 2(\mathrm{G} 11)$ & Em18 & \\
\hline Amebosis $(n=19)$ & 0 & 0 & 0 & 1 & 6 & 0 & 1 & 0 & 0 & 1 & 0 \\
\hline Cysticercosis $(n=19)$ & 11 & 13 & 7 & 9 & 10 & 14 & 12 & 14 & 0 & 0 & 0 \\
\hline Fasciolosis $(n=16)$ & 6 & 4 & 2 & 8 & 10 & 10 & 3 & 10 & 0 & 0 & 0 \\
\hline Filarioses $(n=8)$ & 2 & 2 & 2 & 5 & $8^{\mathrm{b}}$ & 5 & 3 & 5 & 0 & 0 & 0 \\
\hline Schistosomosis $(n=18)$ & 4 & 2 & 2 & 4 & 7 & 2 & 2 & 2 & 0 & 0 & 0 \\
\hline Total $(n=80)$ & 23 & 21 & $13^{\mathrm{b}}$ & 27 & $41^{\mathrm{b}}$ & 31 & 21 & 31 & $0^{\mathrm{a}, \mathrm{b}}$ & $1^{\mathrm{a}, \mathrm{b}}$ & $0^{\mathrm{a}, \mathrm{b}}$ \\
\hline
\end{tabular}

${ }^{a}$ Significantly fewer cross-reactions compared to all other antigens (McNemar test)

b Significant difference in number of cross-reactions compared to EgHF as the gold standard (McNemar test) 
Table 4 Likelihood ratios and post-test probabilities for serological diagnosis of AE or CE

\begin{tabular}{|c|c|c|c|c|c|c|}
\hline \multirow[t]{3}{*}{ Antigen } & \multicolumn{2}{|l|}{ Positive likelihood ratios ${ }^{\mathrm{a}}$} & \multicolumn{4}{|c|}{ Post-test probabilities (\%) } \\
\hline & \multirow[t]{2}{*}{$\begin{array}{l}\text { Alveolar } \\
\text { echinococcosis }\end{array}$} & \multirow[t]{2}{*}{$\begin{array}{l}\text { Cystic } \\
\text { echinococcosis }\end{array}$} & \multirow{2}{*}{$\begin{array}{l}\text { Setting A: } \\
\text { differential diagnosis } \\
\text { of AE from liver } \\
\text { neoplasias (pre-test } \\
\text { probability }=1.5 \%)^{\mathrm{b}}\end{array}$} & \multicolumn{2}{|c|}{$\begin{array}{l}\text { Setting B: differential diagnosis } \\
\text { of CE from cystic liver lesions (pre- } \\
\text { test probability }=1 \%)^{\mathrm{b}}\end{array}$} & \multirow{2}{*}{$\begin{array}{l}\text { Setting C: } \\
\text { screening for AE } \\
\text { in Switzerland } \\
\text { (pre-test } \\
\text { probability } \\
=0.04 \% \text { ) }\end{array}$} \\
\hline & & & & Single test & $\begin{array}{l}\text { With confirmatory } \\
\text { test (AgB-EITB) }\end{array}$ & \\
\hline $\mathrm{EgHF}$ & $51.0(51.0-26.0)$ & $30.0(30.0-15.5)$ & $42.9(27.7-42.9)$ & $23.3(13.5-23.3)$ & $86.45(86.4-76.2)$ & $0.1(0.0-0.1)$ \\
\hline $\mathrm{EgP}$ & $1,342.1(1342.1-671.6)$ & $815.8(815.8-408.4)$ & $95.2(90.8-95.2)$ & $89.2(80.5-89.2)$ & $86.45(86.4-76.2)$ & $0.2(0.1-0.2)$ \\
\hline $\mathrm{EgPI}$ & $1,342.1(1342.1-671.6)$ & $684.2(684.2-342.6)$ & $95.2(90.8-95.2)$ & $87.4(77.6-87.4)$ & $85.40(85.4-74.5)$ & $0.2(0.1-0.2)$ \\
\hline $\mathrm{AgB}$ & $17.0(17.0-9.0)$ & $10.0(10.0-5.5)$ & $20.0(11.7-20.0)$ & $9.2(5.3-9.2)$ & $\mathrm{n} / \mathrm{a}$ & $0.1(0.0-0.1)$ \\
\hline $\mathrm{EmC}$ & $1,342.1(1342.1-671.6)$ & $763.2(763.2-382.1)$ & $95.2(90.8-95.2)$ & $\mathrm{n} / \mathrm{a}$ & $\mathrm{n} / \mathrm{a}$ & $0.1(0.0-0.1)$ \\
\hline EmVF & $51.0(51.0-26.0)$ & $30.0(30.0-15.5)$ & $42.9(27.7-42.9)$ & $\mathrm{n} / \mathrm{a}$ & $\mathrm{n} / \mathrm{a}$ & $0.1(0.0-0.1)$ \\
\hline $\mathrm{EmP}$ & $1,342.1(1342.1-671.6)$ & $763.2(763.2-382.1)$ & $95.2(90.8-95.2)$ & $\mathrm{n} / \mathrm{a}$ & $\mathrm{n} / \mathrm{a}$ & $2.2(1.1-2.2)$ \\
\hline EmPI & $1,342.1(1342.1-671.6)$ & $710.5(710.5-355.8)$ & $95.2(90.8-95.2)$ & $\mathrm{n} / \mathrm{a}$ & $\mathrm{n} / \mathrm{a}$ & $2.2(1.1-2.2)$ \\
\hline Em18 & $1,236.8(1236.8-618.9)$ & $\mathrm{n} / \mathrm{a}$ & $94.8(90.1-94.8)$ & $\mathrm{n} / \mathrm{a}$ & $\mathrm{n} / \mathrm{a}$ & $2.0(1.0-2.0)$ \\
\hline Em2G11 & $1,184.2(1184.2-592.6)$ & $\mathrm{n} / \mathrm{a}$ & $94.6(89.7-94.6)$ & $\mathrm{n} / \mathrm{a}$ & $\mathrm{n} / \mathrm{a}$ & $1.9(1.0-1.9)$ \\
\hline $\begin{array}{l}\text { AgB- } \\
\text { EITB }\end{array}$ & $1,342.1(1342.1-671.6)$ & $657.9(657.9-329.4)$ & $95.2(90.8-95.2)$ & $39.7(24.8-39.7)$ & $\mathrm{n} / \mathrm{a}$ & \\
\hline
\end{tabular}

$A E$ Alveolar echinococcosis, $C E$ cystic echinococcosis

Negative post-test probabilities are not included, as they are all $<0.01 \%$ without significant differences. Post-test probabilities for confirmatory testing of $\mathrm{AE}$ cases are not shown as they are equal to the corresponding values of the confirmatory antigens. Negative likelihood ratios for AE (all antigens) were $<0.0(0.0-0.1)$ and those for CE were $<0.0(0.0-0.1)$ to $0.2(0.1-0.3)$

${ }^{\text {a }}$ Sensitivities and specificities of $99.9 \%$ were used for the calculation of likelihood ratios

b Specificity of sera from patients suffering from non-parasitic liver diseases was used for the calculation

c Specificity of sera from Swiss blood donors was used for the calculation

Diagnostic performance: likelihood ratios and post-test probabilities

Positive and negative likelihood ratios and eventually posttest probabilities (PTPs) were calculated for different clinical and epidemiological settings as described in the "Materials and methods". Negative likelihood ratios for all antigens in terms of $\mathrm{AE}$ or $\mathrm{CE}$ diagnosis were $<0.02$. Thus, negative PTPs were determined to be $<0.01 \%$ in the differential diagnosis and screening in all cases (data not shown). Likelihood ratios and positive PTPs are summarised in Table 4. For the differential diagnosis of the most common liver neoplasias in Switzerland relative to $\mathrm{AE}$, positive PTPs ranged from 20.0 (95\% CI 11.7-20.0, AgBELISA) to $95.2 \%(95 \%$ CI $90.8-95.2 \%$, in several antigens). With the application of confirmatory testing, positive PTPs for Em2G11, Em18 and the combination of both tests were 94.6 (95\% CI 89.7-94.6), 94.8 (95\% CI 90.1-94.8) and $95.1 \%$ (95\% CI 90.7-95.1\%), respectively, without significant differences. The differential diagnosis of cystic lesions compared to CE by the use of E. granulosus-derived antigens in Switzerland showed positive PTPs ranging from 9.2 (95\% CI 5.3-9.2, $\mathrm{AgB})$ to $89.2 \%$ (95\% CI 80.5-89.2\%; EgP). Positive PTPs for AE screening in Switzerland ranged from 0.1 (95\% CI $<0.1-0.1, \mathrm{EgHF}$ ) to $2.2 \%$ (95\% CI 1.1-2.2\%; EmPI, Em18).

Impact of different preparation-techniques

Pearson correlation coefficients for $\mathrm{OD}_{405}$ values were calculated for antigens derived from different morphological correlates (EmC vs. EmP: 0.774; EmC vs. EmVF, EmP vs. EmPI: 0.893; EgP vs. EgPI: 0.822), and all evaluated pairs were significantly correlated.

The cyst/Em2-depleted protoscolex antigen (EmP)based assay showed significantly fewer cross-reactions (19/ 80) than a crude metacestode antigen (EmC, 41/80)-based test ( $p=0.05$, McNemar test). On the other hand, preparation of the integument antigen (EmPI) compared to whole protoscolex antigen (EmP) did not significantly change the results. Isolation of E. multilocularis cyst fluid $(\mathrm{EmVF})$ as compared to the whole metacestode antigen (EmC) yielded only a trend in fewer cross-reactions (31/80 and $41 / 80$, respectively, $p=0.08$ ).

Among the E. granulosus-derived antigens, no difference was found between $\mathrm{EgHF}$ and $\mathrm{EgP}$, whereas preparation of the integument (EgPI) as compared to whole 
protoscolex antigen (EgP) had overall fewer cross-reactions (13/80 and 21/80, respectively; $p<0.005$ ), especially for cysticercosis (7/19 and 13/19, respectively; $p=0.03)$. Unfortunately a trend towards a lower sensitivity for $\mathrm{CE}$ was noted in EgPI (81.3\%; EgP: 96.9\%; $p=0.06$ ).

\section{Discussion}

The aim of this study was to compare the diagnostic performances of crude and partially purified native Echinococcus antigens that are easy to produce and to assess their value in defined clinical and epidemiological settings. Typical situations in which specific anti-Echinococcus antibodies are determined are the differential diagnosis of $\mathrm{AE}$ and $\mathrm{CE}$ from other cystic- or tumour-like pathologies and epidemiological surveillance programmes. Therefore, treatment-naïve $\mathrm{AE}$ and $\mathrm{CE}$-patients, patients with other types of liver pathologies as well as a screening situation in a low endemic area were included in the evaluation.

The most commonly used tests for the diagnosis of CE are based on hydatid fluid antigens of E. granulosus. These tests have relatively high sensitivities for hepatic (85-95\%) and multiple organ cysts (90-100\%), but lower sensitivities for lung cysts (50-60\%) [33-35]. Additionally, tests based on the partially purified lipoproteins antigen $\mathrm{B}(\mathrm{AgB})$ and antigen 5 (both found in the germinal membrane, protoscolex parenchyma and cyst fluid [36]) are used either in ELISA or immunoblot assays. The major drawback of these tests is the lack of species specificity, as cross-reactions between $\mathrm{AE}$ and $\mathrm{CE}$ and many other helminthic infections are commonly observed (cestodes, $89 \%$; nematodes, 39\%; trematodes; 30\%) [5, 20, 37-40]. Assays based on a recombinant subunit of $\mathrm{AgB}$, denoted $\operatorname{rAgB} 8 / 2$, showed sensitivities of $45-93 \%$ and specificities of $86-99 \%$ with the same cross-reactions as mentioned above [20, 41, 42].

In our study, all non-AE-derived native or partially purified antigens exhibited sensitivities ranging from 81.3 to $96.9 \%$ for the diagnosis of CE; as such, they are not significantly different from antigen preparations that are currently available. As only seven of the $32 \mathrm{CE}$ patients evaluated had no hepatic involvement in our study, no sound conclusions on test performances in cases with extrahepatic lesions can be drawn. Additionally, cysts of morphology type CL, based on the WHO classification system, cannot be distinguished from hepatic cysts of other origins by US, and differential diagnosis in these cases is especially difficult [21]. Unfortunately, no patients with cysts of this type were available for the study. Thus, the relatively high sensitivities for CE may be biased by the panel of sera used and may not be extrapolated for the serological diagnosis of these types of lesions.
Different antigen preparations are in use for the serological diagnosis of AE. The most commonly used tests, although not species specific, are also ELISA based on E. granulosus hydatid fluid (EgHF) and have sensitivities ranging from 90 to $97 \%$ for AE [40]. For the detection of $\mathrm{AE}$ cases, the native antigens tested in our study, regardless of the species they were derived from, proved to be well suited, as all cases were detected by all tests. Our antigens exhibited high sensitivities of $>99 \%$ for the diagnosis of $\mathrm{AE}$, but species specificity was lacking.

For species-specific serological diagnosis, recombinant E. multilocularis antigens (such as II/3-10, Em10- or Em18-antigen; all of which belong to the same protein family), either alone or in the form of the Em2Plus ELISA (Bordier Affinity Products, Crissier, Switzerland) as a combination of the II/3-10 and Em2 antigens, are commonly used. These antigens have diagnostic sensitivities ranging between 81 and $100 \%$ and discriminate between $\mathrm{AE}$ and $\mathrm{CE}$ with a reliability ranging from 80 to $\geq 95 \%[15$, $26,27,43-46]$. The native and partially purified antigens tested in our study did not discriminate between $\mathrm{AE}$ and CE: they all recognised all $\mathrm{AE}$ and at least $81.3 \%$ of $\mathrm{CE}$ cases. As already well-established, only the Em2G11- and Em18-antigens in our antigen panel differentiated AE from $\mathrm{CE}$ in 81.3 and $93.7 \%$ of cases, respectively, thereby providing tools for species differentiation.

The various AgB-ELISA that are in use for the diagnosis of either $\mathrm{CE}$ or $\mathrm{AE}$ have been found to have different sensitivities. Mamuti and colleagues [47] used a recombinant $\mathrm{AgB} 8 / 1$, reporting sensitivities of $88.0 \%$ (95\% CI $75.7-95.5 \%$ ) for $\mathrm{CE}$ and $37.8 \%$ (95\% CI $23.7-53.5 \%$ ) for AE. In another study by Li and colleagues [48], a recombinant $\mathrm{AgB}$ subunit 8, basically prepared as described by Mamuti and colleagues [47], reached sensitivities of 77.6\% (95\% CI $77.6-82.7 \%)$ for $\mathrm{CE}$ and $85.0 \%$ (95\% CI $78.8-89.9 \%$ ) for $\mathrm{AE}$; in terms of $\mathrm{AE}$, this is significantly more sensitive than that reached by Mamuti and colleagues with the recombinant $\mathrm{AgB} 8 / 1$, but not more sensitive than that found in our study using native $\mathrm{AgB}$ ( $>99.9 \%$; $95 \% \mathrm{CI}$ 94.3-100\%). Sensitivities for CE did not differ significantly between all three studies and were comparable with the results of a study by Gihan and colleagues [49] where a sensitivity of $82.5 \%$ (95\% CI $73.2-95.8 \%$ ) was reported. The differences in the sensitivities for AE are likely to be explained as being related to the different panels of sera used. Whereas Mamuti and colleagues evaluated sera from patients under treatment or post-surgery, in the study by $\mathrm{Li}$ and colleagues and in our study only sera from treatmentnaïve patients were used, leading to distinctly higher sensitivities.

The parallel use of multiple native antigens (EgHF, EgP, $\mathrm{AgB}$ and Em2) in a dot-immunogold filtration assay (DIGFA) analysing patients with probable or confirmed CE 
or $\mathrm{AE}$ yielded sensitivities for $\mathrm{CE}$ and $\mathrm{AE}$ of 80.7 and $92.9 \%$, respectively, with a specificity of $89.6 \%$ [50]. The isolated analysis of the AgB-dot had a sensitivity and specificity for CE of 68.4 and $93.4 \%$, respectively, whereas the isolated analysis of the Em2-dot diagnosed $83.3 \%$ of AE patients with a specificity of $90.3 \%$ and reliability for species differentiation of $83.0 \%$ [50]. Although the overall performances of these tests were lower than those in our study, differences in the epidemiological background and serum panel used have to be taken into account.

Test specificities were excellent for most of our antigens when Swiss patients with non-parasitic liver lesions were tested. False positive reactions were only recorded for the AgB-, EgHF- and EmVF-based ELISA. The application of our confirmatory tests subsequently removed all of these false-positive reactions, as these tests have specificities $>99.9 \%$. Except for the Em2(G11) and the Em18 antigen, all antigens used in the ELISA showed a substantial number of cross-reactions, mainly with antibodies in sera of patients suffering from cysticercosis and fasciolosis, although the intensity of the serological reactions (given in Fig. 1) varied. However, cut-off-values could be adapted for certain antigens (mainly EmP, EmVF) to maintain a high sensitivity for AE while minimising the number of cross-reactions (data not shown). Nevertheless, additional evaluations by confirmatory testing and imaging studies should be performed. Fasciolosis in the hepatic stage can be differentiated by clinical symptoms and typical radiological signs, such as clustered hepatic micro-abscesses in a serpentine-fashion or the "tunnels-and-caves-sign" [51, 52]. In addition, species-specific tests based on excretory/ secretory-proteins of Fasciola sp. allow discrimination by serology-for example, by the Fas2-ELISA [53]. Further diagnosis could be achieved by coproscopy, i.e., demonstrating eggs of the parasite in stool samples [52, 54]. For the differential diagnosis of cysticercosis, a highly specific western blot based on Taenia solium-derived glycoproteins and an ELISA based on recombinant antigens are available [55, 56], although in EITB cross reactivity with sera from $\mathrm{AE}$ patients with non-diagnostic higher molecular weight components have been reported [57]. Schistosomosis in imaging studies mainly appears in the periportal tissue together with fibrosis, calcification (mainly in Schistosoma japonicum, rare in S. mansoni) and thickening of the walls of periportal septae and the liver capsule [58], features usually not seen in Echinococcus patients. Diagnosis is usually achieved by the detection of the parasite's eggs in stool or urine, except for the acute phase, which is clinically very distinct from echinococcosis [59]. Thus, other parasitic diseases with manifestation in the liver can be distinguished by means of the clinical presentation, direct detection of parasite's eggs, serology and radiology. In addition, by the application of confirmatory tests, all but one cross-reaction to antibodies in the serum of a patient suffering of amoebic liver abscess (initial test: EmCELISA; confirmation test: Em18-ELISA) were prevented.

Despite rising incidences in many regions, $\mathrm{AE}$ and $\mathrm{CE}$ prevalences are still low compared to other hepatic affections such as liver cysts or hepatic neoplasias [60]. Thus, it is crucial in practical clinical work to be able to estimate the probability that a patient is suffering from $\mathrm{AE}$ or $\mathrm{CE}$ once the serological test results are known. The calculation of positive or negative PTPs in a given patient or collective patients using likelihood ratios that are based on test sensitivities and specificities, together with the use of the pretest probability as an estimator that integrates the prevalence of a disease among the people tested and additional clinical and radiological information, is one of the best tools for this task [30].

In the case of screening campaigns, only the anticipated prevalence is determined-no further information on the people tested is known. Therefore, the pre-test probability is low and essentially in accordance with AE or CE prevalence. Hence, PTPs are low in such settings. In the Swiss example (screening for AE), the positive PTPs are $<0.1 \%$, and they do not improve considerably with the application of confirmatory tests $(<2.2 \%)$. In contrast, the corresponding negative PTPs are all $<0.01$; consequently, they allow an infection to be excluded with a high probability. However, a substantial number of patients might be missed in large screening programmes, as has been outlined by Torgerson and Deplazes [61]. This clearly underlines the necessity of additional examination techniques (i.e. imaging techniques) to identify and confirm cases in such settings. A drawback of our assumption is the fact that the number of patients with died-out lesions is hard to estimate. Therefore, these patients could not be included in the estimated prevalence. However, patients prone to develop clinically relevant disease within $0-15$ years post-screening were covered. Thus, the estimated prevalence may not exactly depict the prevalence, but the dimension is appropriately covered.

In contrast, in defined clinical settings where highly preselected patients based on clinical and radiological information are tested, $\mathrm{CE}$ and $\mathrm{AE}$ prevalences are substantially higher than those found in the general population. The same may be true for combined radiological and serological screening programmes. The estimated prevalence of AE lesions among Swiss patients with suspicion of liver neoplasias undergoing imaging examinations was $1.5 \%$, and this value was taken as the pre-test probability. Therefore, positive PTPs of all tests in combination with confirmatory testing were $>94.6 \%$. The estimated CE prevalence among patients at the University Hospital of Zurich with cystic liver lesions was $1 \%$. In this clinical setting, positive PTPs of the tests based on E. granulosus- 

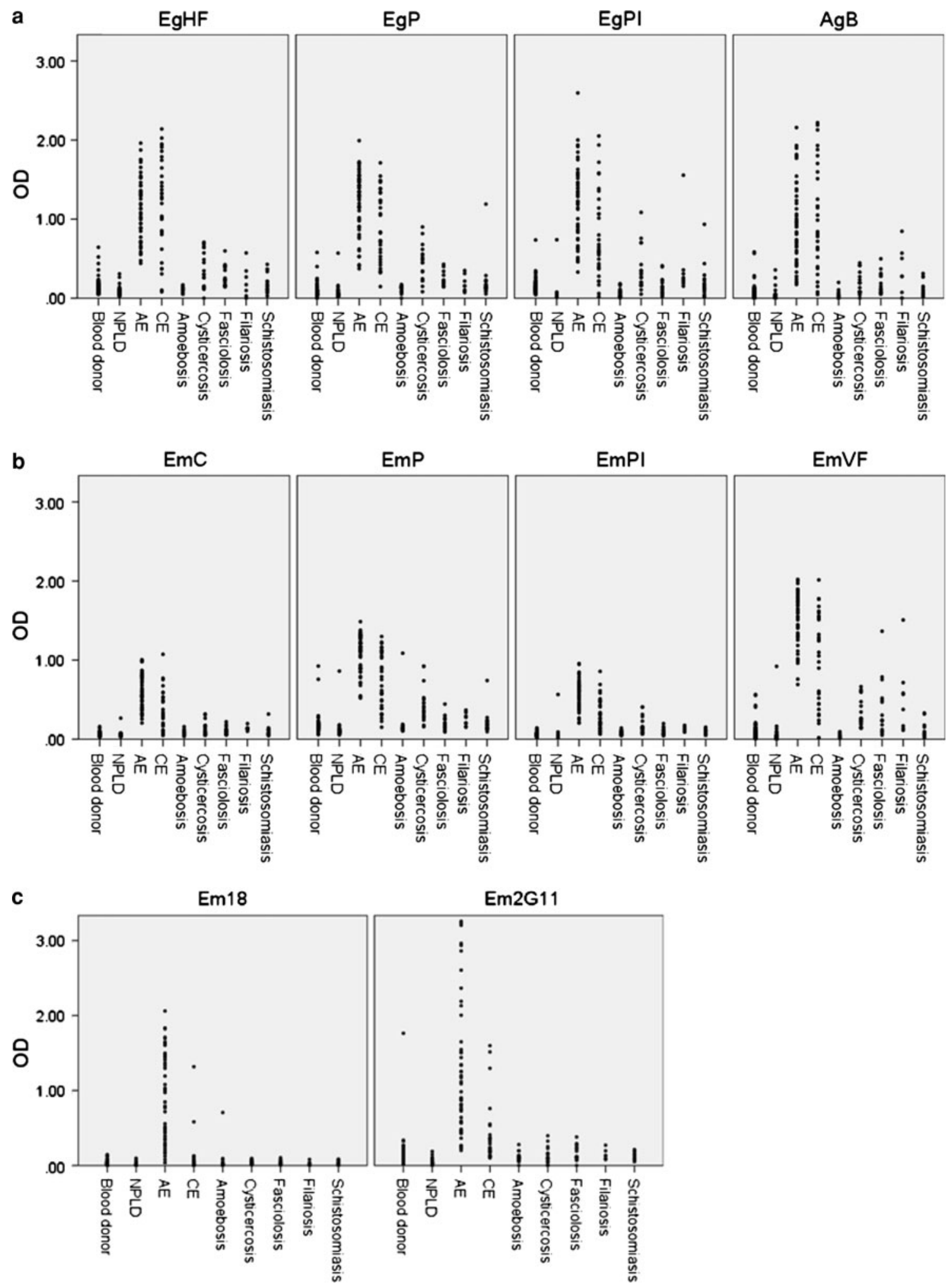

Fig. 1 Intensity of serological reaction $\left(\mathrm{OD}_{405}\right)$ to defined patient groups of different metacestode-derived antigens of Echinococcus granulosus (a) and E. multilocularis (b) in comparison to the species- specific confirmatory antigens Em18 and Em2G11 (c). AE Alveolar echinococcosis, $C E$ cystic echinococcosis, $N P L D$ non-parasitic liver disease. See Table 1 for definitions of antigens 
derived antigens ranged from 9.2 (AgB-ELISA) to $89.2 \%$ (EgP). The AgB-EITB test results for confirming these values were around $87 \%$ for all tests. In both settings, serology contributed substantially to ascertain the definite diagnosis.

Other diagnostic evaluations and clinical presentations will contribute to the pre-test-probabilities and therefore significantly change PTPs. Several cyst stages in CE have pathognomonic radiological signs [21]. In $\mathrm{AE}$, specific radiological features, such as the lack of perilesional enhancement as compared to neoplasias [62], will restrict differential diagnoses and therefore influence pre-test and subsequently PTPs in a positive or negative way. For example, if the differentiation of $\mathrm{CE}$ from other cystic liver lesions were possible with a probability of $75 \%$, positive PTPs for CE would increase to $>95 \%$ after serological diagnosis with EgPI as compared to $87 \%$ when only prevalence is used. However, one has to be aware that even minor changes in the specificity of any test will result in a considerable change in the PTP.

Depletion of Em2 and adhering cyst-material in E. multilocularis-derived antigens (EmP, EmPI) neither reduced the number of cross-reactions with antibodies in sera from patients suffering from other parasitic infections nor improved test sensitivities or specificities. Also, the isolation of integument antigens (EgPI; EmPI) as compared to whole protoscolex antigens (EgP; EmP) had no significant impact on the diagnostic performances, although crossreactions were significantly lower in the EgPI-based assay. The same holds true for the purification of protoscoleces of E. multilocularis as compared to crude metacestodederived antigens $(\mathrm{EmC})$, where the number of cross-reactions could be significantly decreased.

In conclusion, different easy-to-prepare and inexpensive metacestode-derived native antigens of E. multilocularis and $E$. granulosus are valuable tools for the diagnosis of $\mathrm{CE}$ and $\mathrm{AE}$ in clinical settings. Negative results in any of these tests rule out $\mathrm{AE}$ cases with a high certainty, and due to the high sensitivities, hardly any clinical cases would be missed. As only a limited number of inactive CE cases or cases with isolated lung cysts were available for this study, the same conclusion might not automatically apply for CE patients.

Based on the tests evaluated here, an efficient approach to the serological diagnosis of echninococcosis is primary testing with the crude E. granulosus protoscolex-derived antigen $(\mathrm{EgP})$ alone or in combination with EgHF, followed by the additional testing of positive cases with species-specific antigens Em18 and Em2G11 for differential diagnosis at species level and confirmation of AE. Sera testing negative in the $\mathrm{AE}$-specific tests are suspicious for $\mathrm{CE}$ and should be confirmed by, for example, AgB-EITB.
Conflict of interest None.

\section{References}

1. Eckert J, Deplazes P, Kern P. Alveolar Echinococcosis (Echinococcus multilocularis) and other forms of echinococcosis (Echinococcus vogeli and Echinococcus oligarthrus). In: Brown D, Palmer SR, Torgerson PR, Soulsby EJL, editors. Zoonoses. Oxford: Oxford University Press; 2011. p. 671-701.

2. Brunetti E, Kern P, Vuitton DA, Writing Panel for the WHO Informal Working Group on Echinococcosis. Expert consensus for the diagnosis and treatment of cystic and alveolar echinococcosis in humans. Acta Trop. 2010;114:1-16.

3. Ammann RW, Eckert J. Cestodes. Echinococcus. Gastroenterol Clin North Am. 1996;25:655-89.

4. Torgerson PR, Schweiger A, Deplazes P, Pohar M, Reichen J, Ammann RW, et al. Alveolar echinococcosis: from a deadly disease to a well-controlled infection. Relative survival and economic analysis in Switzerland over the last 35 years. J Hepatol. $2008 ; 49: 72-7$.

5. Eckert J, Deplazes P. Biological, epidemiological, and clinical aspects of echinococcosis, a zoonosis of increasing concern. Clin Microbiol Rev. 2004;17:107-35.

6. Schweiger A, Ammann RW, Candinas D, Clavien PA, Eckert J, Gottstein B, et al. Human alveolar echinococcosis after fox population increase, Switzerland. Emerg Infect Dis. 2007;13: 878-82.

7. Bruzinskaite R, Marcinkute A, Strupas K, Sokolovas V, Deplazes P, Mathis A, et al. Alveolar echinococcosis, Lithuania. Emerg Infect Dis. 2007;13:1618-9.

8. Torgerson PR, Karaeva RR, Corkeri N, Abdyjaparov TA, Kuttubaev OT, Shaikenov BS. Human cystic echinococcosis in Kyrgystan: an epidemiological study. Acta Trop. 2003;85:51-61.

9. Vuitton DA, Zhou H, Bresson-Hadni S, Wang Q, Piarroux M, Raoul F, et al. Epidemiology of alveolar echinococcosis with particular reference to China and Europe. Parasitology. 2003;127: S87-107.

10. Ito A, Romig T, Takahashi K. Perspective on control options for Echinococcus multilocularis with particular reference to Japan. Parasitology. 2003;127:S159-72.

11. Sako Y, Nakao M, Nakaya K, Yamasaki H, Ito A. Recombinant antigens for serodiagnosis of cysticercosis and echinococcosis. Parasitol Int. 2006;55:S69-73.

12. Coudert J, Ambroise-Thomas P, Kien Truong T, Pothier MA. 1st results concerning the serologic diagnosis of hydatid cyst by a new technic of immunofluorescence on slides. Bull Soc Pathol Exot Filiales. 1967;60:555-63.

13. Auer H, Hermentin K, Aspock H. Demonstration of a specific Echinococcus multilocularis antigen in the supernatant of in vitro maintained protoscolices. Zentralbl Bakteriol Mikrobiol Hyg A. 1988;268:416-23.

14. Gottstein B, Eckert J, Fey H. Serological differentiation between Echinococcus granulosus and E. multilocularis infections in man. Z Parasitenkd. 1983;69:347-56.

15. Deplazes P, Gottstein B. A monoclonal antibody against Echinococcus multilocularis Em2 antigen. Parasitology. 1991;103:41-9.

16. Gottstein B, Deplazes P, Aubert M. Echinococcus multilocularis: immunological study on the "Em2-positive" laminated layer during in vitro and in vivo post-oncospheral and larval development. Parasitol Res. 1992;78:291-7.

17. Gottstein B, Eckert J, Michael SA, Thompson RCA. Echinococcus granulosus antigens: Immunoelectrophoretic and Western Blot analysis of hydatid cyst fluids. Parasitol Res. 1987;73:186-9. 
18. Frayha GJ, Bahr GM, Haddad R. The lipids and phospholipids of hydatid protoscolices of Echinococcus granulosus (Cestoda). Int J Parasitol. 1980;10:213-6.

19. Frayha GJ, Haddad R. Comparative chemical composition of protoscolices and hydatid cyst fluid of Echinococcus granulosus (Cestoda). Int J Parasitol. 1980;10:359-64.

20. Lorenzo C, Ferreira HB, Monteiro KM, Rosenzvit M, Kamenetzky L, Garcia HH, et al. Comparative analysis of the diagnostic performance of six major Echinococcus granulosus antigens assessed in a double-blind, randomized multicenter study. J Clin Microbiol. 2005;43:2764-70.

21. MacPherson CNL, Vuitton DA, Gharbia HA, Caremani M, Frider $\mathrm{B}$, Brunetti E, WHO informal working group on the development of a standardised classification for CE by US. International classification of ultrasound images in cystic echinococcosis for application in clinical and field epidemiological settings. Acta Trop. 2003;85:253-61.

22. Eckert J, Pohlenz J. On the effect of mebendazole on metacestodes of Mesocestoides corti and Echinococcus multilocularis (author's translation). Tropenmed Parasitol. 1976;27:247-62.

23. Muller N, Frei E, Nunez S, Gottstein B. Improved serodiagnosis of alveolar echinococcosis of humans using an in vitro-produced Echinococcus multilocularis antigen. Parasitology. 2007;134: 879-88.

24. Oriol R, Williams JF, Perez Esandi MV, Oriol C. Purification of lipoprotein antigens of Echinococcus granulosus from sheep hydatid fluid. Am J Trop Med Hyg. 1971;20:569-74.

25. Ortona E, Rigano R, Margutti P, Notargiacomo S, Ioppolo S, Vaccari S, et al. Native and recombinant antigens in the immunodiagnosis of human cystic echinococcosis. Parasite Immunol. 2000;22:553-9.

26. Ito A, Osawa $\mathrm{Y}$, Nakao M, Horii T, Okamoto M, Itoh M, et al. Em18 and Em16, new serologic marker epitopes for alveolar echinococcosis in western blot analysis, are the only two epitopes recognized by commercially available weak positive (cut off) sera for Em2plus-ELISA. J Helminthol. 1995;69:369-71.

27. Ito A, Xiao N, Liance M, Sato MO, Sako Y, Mamuti W, et al. Evaluation of an enzyme-linked immunosorbent assay (ELISA) with affinity-purified Em18 and an ELISA with recombinant Em18 for differential diagnosis of alveolar echinococcosis: results of a blind test. J Clin Microbiol. 2002;40:4161-5.

28. Akobeng AK. Understanding diagnostic tests 3: receiver operating characteristic curves. Acta Paediatr. 2007;96:644-7.

29. Greiner M, Sohr D, Gobel P. A modified ROC analysis for the selection of cut-off values and the definition of intermediate results of serodiagnostic tests. J Immunol Methods. 1995;185: $123-32$.

30. Akobeng AK. Understanding diagnostic tests 2: likelihood ratios, pre- and post-test probabilities and their use in clinical practice. Acta Paediatr. 2007;96:487-91.

31. Leporrier J, Maurel J, Chiche L, Bara S, Segol P, Launoy G. A population-based study of the incidence, management and prognosis of hepatic metastases from colorectal cancer. Br J Surg. 2006;93:465-74

32. Deplazes P, Grimm F, Sydler T, Tanner I, Kapel CM. Experimental alveolar echinococcosis in pigs, lesion development and serological follow up. Vet Parasitol. 2005;130:213-22.

33. Siracusano A, Bruschi F. Cystic echinococcosis: progress and limits in epidemiology and immunodiagnosis. Parassitologia. 2006;48:65-6.

34. Ito A, Craig PS. Immunodiagnostic and molecular approaches for the detection of taeniid cestode infections. Trends Parasitol. 2003;19:377-81.

35. Siles-Lucas M, Gottstein B. Molecular tools for the diagnosis of cystic and alveolar echinococcosis. Trop Med Int Health. 2001;6:463-75.
36. Sanchez F, March F, Mercader M, Coll P, Munoz C, Prats G. Immunochemical localization of major hydatid fluid antigens in protoscoleces and cysts of Echinococcus granulosus from human origin. Parasite Immunol. 1991;13:583-92.

37. Gottstein B, Lengeler C, Bachmann P, Hagemann P, Kocher P, Brossard M, et al. Sero-epidemiological survey for alveolar echinococcosis (by Em2-ELISA) of blood donors in an endemic area of Switzerland. Trans R Soc Trop Med Hyg. 1987;81:9604.

38. Craig PS, Giraudoux P, Shi D, Bartholomot B, Barnish G, Delattre $\mathrm{P}$, et al. An epidemiological and ecological study of human alveolar echinococcosis transmission in south Gansu, China. Acta Trop. 2000;77:167-77.

39. Zhang W, Li J, McManus DP. Concepts in immunology and diagnosis of hydatid disease. Clin Microbiol Rev. 2003;16:18-36.

40. Rafiei A, Craig PS. The immunodiagnostic potential of protoscolex antigens in human cystic echinococcosis and the possible influence of parasite strain. Ann Trop Med Parasitol. 2002;96: 383-9.

41. Rott MB, Fernandez V, Farias S, Ceni J, Ferreira HB, Haag KL, et al. Comparative analysis of two different subunits of antigen B from Echinococcus granulosus: gene sequences, expression in Escherichia coli and serological evaluation. Acta Trop. 2000; 75:331-40.

42. Virginio VG, Hernandez A, Rott MB, Monteiro KM, Zandonai $\mathrm{AF}$, Nieto A, et al. A set of recombinant antigens from Echinococcus granulosus with potential for use in the immunodiagnosis of human cystic hydatid disease. Clin Exp Immunol. 2003;132: 309-15.

43. Jiang L, Wen $\mathrm{H}$, Ito A. Immunodiagnostic differentiation of alveolar and cystic echinococcosis using ELISA test with 18-kDa antigen extracted from Echinococcus protoscoleces. Trans R Soc Trop Med Hyg. 2001;95:285-8.

44. Gottstein B, Jacquier P, Bresson-Hadni S, Eckert J. Improved primary immunodiagnosis of alveolar echinococcosis in humans by an enzyme-linked immunosorbent assay using the Em2plus antigen. J Clin Microbiol. 1993;31:373-6.

45. Frosch PM, Frosch M, Pfister T, Schaad V, Bitter-Suermann D. Cloning and characterisation of an immunodominant major surface antigen of Echinococcus multilocularis. Mol Biochem Parasitol. 1991;48:121-30.

46. Xiao N, Mamuti W, Yamasaki H, Sako Y, Nakao M, Nakaya K, et al. Evaluation of use of recombinant Em18 and affinity-purified Em18 for serological differentiation of alveolar echinococcosis from cystic echinococcosis and other parasitic infections. J Clin Microbiol. 2003;41:3351-3.

47. Mamuti W, Yamasaki H, Sako Y, Nakao M, Xiao N, Nakaya K, et al. Molecular cloning, expression, and serological evaluation of an 8-kilodalton subunit of antigen B from Echinococcus multilocularis. J Clin Microbiol. 2004;42:1082-8.

48. Li T, Ito A, Chen X, Sako Y, Qiu J, Xiao N, et al. Specific IgG responses to recombinant antigen $\mathrm{B}$ and Em18 in cystic and alveolar echinococcosis in China. Clin Vaccine Immunol. 2010; $17: 470-5$.

49. Tawfeek GM, Elwakil HS, El-Hoseiny L, Thabet HS, Sarhan RM, Awad NS, et al. Comparative analysis of the diagnostic performance of crude sheep hydatid cyst fluid, purified antigen B and its subunit ( $12 \mathrm{Kda})$, assessed by ELISA, in the diagnosis of human cystic echinococcosis. Parasitol Res. 2011;108:371-6.

50. Feng X, Wen H, Zhang Z, Chen X, Ma X, Zhang J, et al. Dot immunogold filtration assay (DIGFA) with multiple native antigens for rapid serodiagnosis of human cystic and alveolar echinococcosis. Acta Trop. 2010;113:114-20.

51. Czermak BV, Akhan O, Hiemetzberger R, Zelger B, Vogel W, Jaschke W, et al. Echinococcosis of the liver. Abdom Imaging. 2008;33:133-43. 
52. Lim JH. Parasitic diseases in the abdomen: imaging findings. Abdom Imaging. 2008;33:130-2.

53. Espinoza JR, Maco V, Marcos L, Saez S, Neyra V, Terashima A, et al. Evaluation of Fas2-ELISA for the serological detection of Fasciola hepatica infection in humans. Am J Trop Med Hyg. 2007;76:977-82.

54. Marcos LA, Terashima A, Gotuzzo E. Update on hepatobiliary flukes: fascioliasis, opisthorchiasis and clonorchiasis. Curr Opin Infect Dis. 2008;21:523-30.

55. Tsang VC, Brand JA, Boyer AE. An enzyme-linked immunoelectrotransfer blot assay and glycoprotein antigens for diagnosing human cysticercosis (Taenia solium). J Infect Dis. 1989;159: $50-9$.

56. Hubert K, Andriantsimahavandy A, Michault A, Frosch M, Muhlschlegel FA. Serological diagnosis of human cysticercosis by use of recombinant antigens from Taenia solium cysticerci. Clin Diagn Lab Immunol. 1999;6:479-82.
57. Ito A, Plancarte A, Ma L, Kong Y, Flisser A, Cho SY, et al. Novel antigens for neurocysticercosis: simple method for preparation and evaluation for serodiagnosis. Am J Trop Med Hyg. 1998;59:291-4.

58. Manzella A, Ohtomo K, Monzawa S, Lim JH. Schistosomiasis of the liver. Abdom Imaging. 2008;33:7.

59. Ross AG, Bartley PB, Sleigh AC, Olds GR, Li Y, Williams GM, et al. Schistosomiasis. N Engl J Med. 2002;346:1212-20.

60. Eckert J, Gemmel MA, Meslin F-X, Pawlowski ZS. WHO/OIE manual on echinococcosis in humans and animals: a public health problem of global concern. Paris: World Organisation for Animal Health and World Health Organization; 2001.

61. Torgerson PR, Deplazes P. Echinococcosis: diagnosis and diagnostic interpretation in population studies. Trends Parasitol. 2009;25:164-70.

62. Reuter S, Nussle K, Kolokythas O, Haug U, Rieber A, Kern P, et al. Alveolar liver echinococcosis: a comparative study of three imaging techniques. Infection. 2001;29:119-25. 